\title{
Comparison between SIFT and SURF image forgery Algorithms
}

\author{
Ajay Sahay \\ Research scholar \\ computer science BBAU \\ University Lucknow \\ Uttar Pradesh India
}

\author{
Anupama Gautam \\ Research scholar computer \\ science BBAU University Lucknow \\ Uttar Pradesh India
}

\begin{abstract}
The adjustment of any processed image that stows away essential knowledge. This kind of advanced footage has arduous to get distinctive piece of processed image. During this paper audits the varied kinds of image falsification recognition technique that is on past explores and additional works.
\end{abstract}

\section{Keywords}

Copy-move, segmentation, key-point extraction,sift,etc.

\section{INTRODUCTION}

Impressive and at once accessible photograph redaction tools like Photoshop and freehanded created manipulating and alter of state of digital photos unit of measurement successful. Because of this increase of the image forgery in virtual and social media. This trend lands up in severe vulnerabilities and loss of quality with within the digital photos. As things go the (SS) Social Security detection of image forgery is vital. throughout this sense image modification of state detection is that the central attraction of digital image forensics.

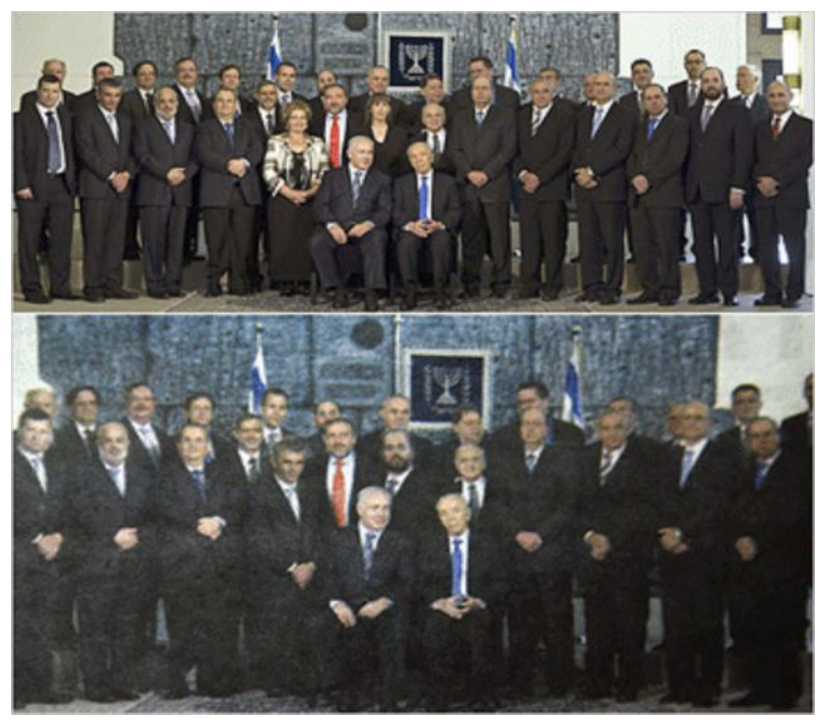

Figure 1:Modifed image in parliament

Copy-move of image may be a important space of image forgery, throughout this a particular apart springs and pasted on the region of same image to cover some crucial data. throughout the these sorts of forgeries, digital image preprocessing techniques like scaling, rotation, blurring, noise and compression, unit of activity applied to create satisfying forgeries.
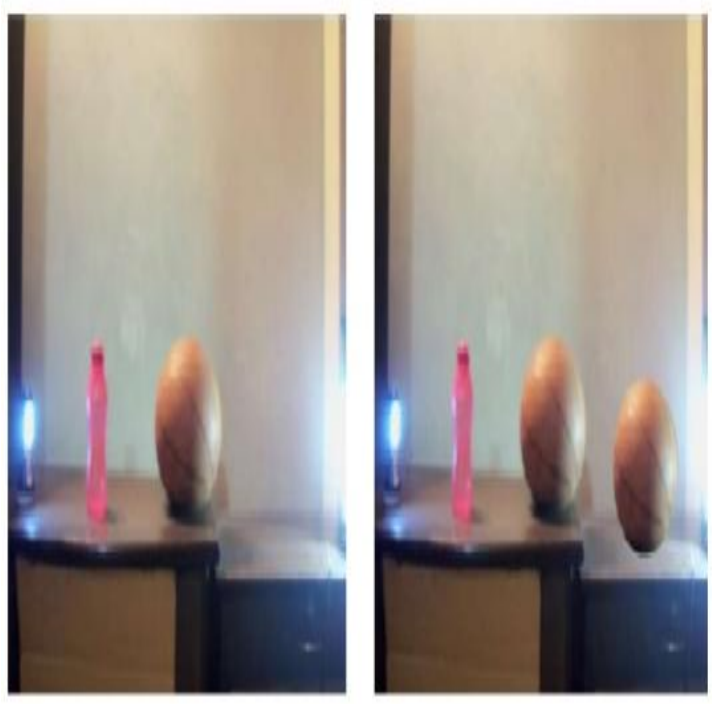

Figure 2: Copy- Move image

Drawback of research those forgeries, considering the person of area-duplication; there are a unit a unit not less than similar areas in the course of and the present techniques, there are two replica-move photograph forgery detection methods block-based totally algorithms ${ }^{1,2}$ and feature keyfactor extraction ${ }^{3,4,5}$ based algorithms. Compute for each block. comparable characteristic points are selected and paired to seek out stable areas.

The photograph is not divided into blocks, the feature vectors ar matched to look out stable areas. .Chi-guy Pun, Xiao-Chen Yuan, and $\mathrm{Xiu}-\mathrm{Li} \mathrm{Bi}^{7}$ proposed a reproduction flow into ikon forgery detection approach as a combination of easy block based mostly approach and key purpose based approach. The adjustive over-segmentation is analogous to block-based all detection strategies inside that to divide the host image into non-overlapping and ir-regular blocks adjustive to the host image texture. just like the key factor-based approach in characteristic-point matching, the characteristic factors square measure extracted from every image block as block alternatives. 


\section{METHODLOGY}

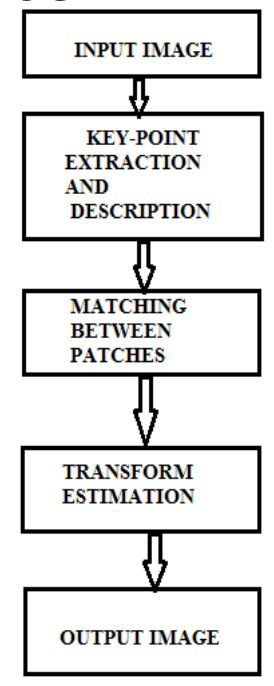

Figure 3: Methodology flow chart

\section{SIFT (SCALE INVARIANT FEATURE TRANSFORMATION)}

SIFT key-point detected and distorted difficult regions from the image supported the sturdy matching methodology. It's smart performance in difficult forgeries with duplicated regions. this will be the one in each of the restrictions for some footage. For the reason that algorithmic program cannot notice dependable key-points. Believe several methods to develop the detection performance like PCA SIFT (Principal part analysis SIFT) or histograms and intrinsic signal data point. In SIFT having 5 steps are

- Scale space Extreme

- $\quad$ Detection Key point

- Localization Orientation

- Assignment Feature

- Description Feature Matching

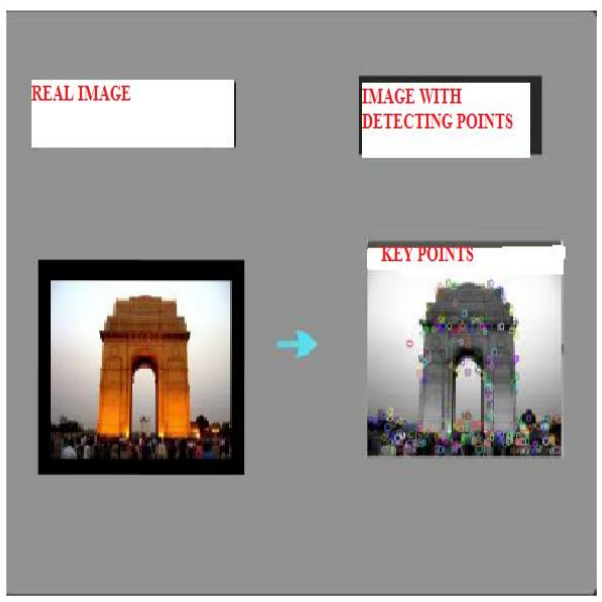

Figure 4: Key point detection using SIFT

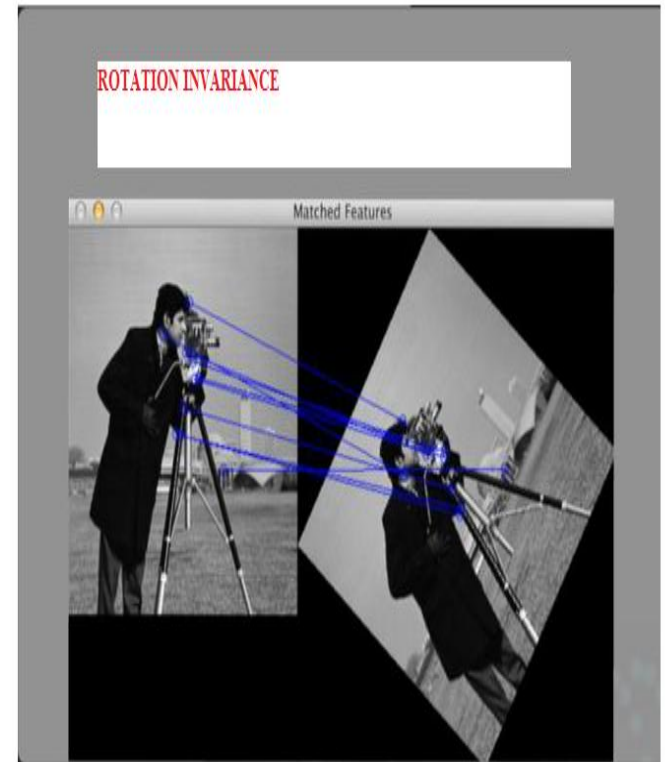

Figure 5: Rotation invariant using SIFT

\section{SEGMENTATION}

To split the cope picture from pasting photograph, this might be divided into little or no patches that square measure semantically harmless to the others.

Every photo is mainly divided into no but 100 patches. to hunt out a forceful detection result would really like not a many form of key-factors. to apply segmentation technique is used to relinquish foal results. The host picture is split into overlapping regular blocks, which may be computationally highlypriced because of the photo length can boom. those algorithms cannot direction huge geometrical differences within the forgery areas. on the grounds that their block method is everyday in kind, their take into account rate is low.

\section{SPEED UP ROBUST FEATURE (SURF)}

Key motive extraction is based on multi-scale area theory and accordingly the detector is predicated on Wellington boot matrix that has wise presentation and correctness. This formulation preliminary identifies picture hobby factors and computes the descriptors for the important thing-factors.

SURF creates a stack whereas not 2:1 down sampling for higher levels inside the pyramid resulting in photographs of identical decision. In key purpose matching step, the nighest neighbor is made public because of the key purpose with minimum geometer distance for the invariant descriptor vector. decrease used a a number of sensible pastime that obtained with the aid of contrast the location of the nearest neighbor there to $2^{\text {nd }}$ closest neighbor. 


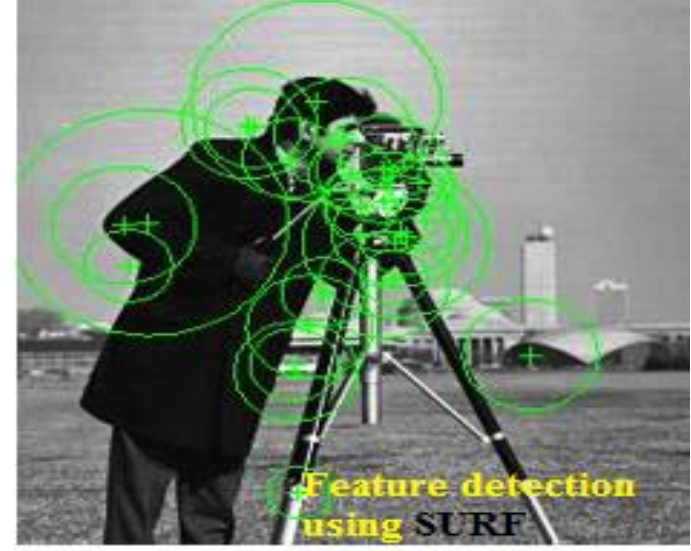

(a)

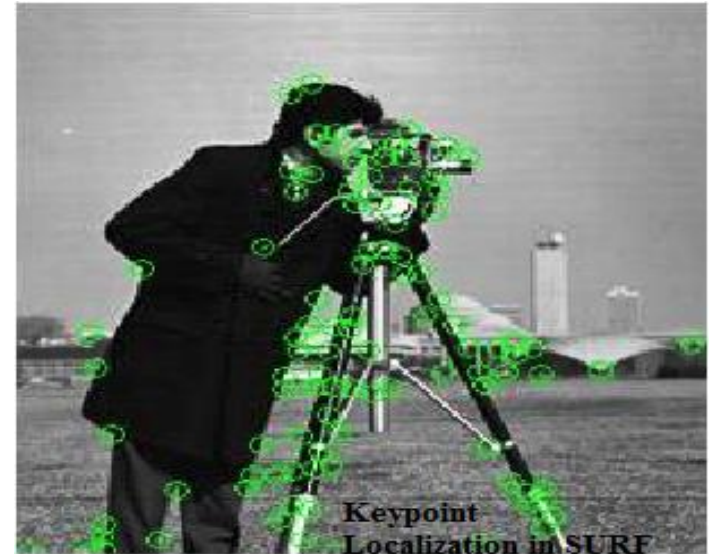

(b)

Figure 6: (a) Feature detection using SURF, (b) Key point Localization using SURF

Table 1 Comparison SIFT and SURF Algorithm

\begin{tabular}{|c|c|c|c|c|c|c|}
\hline ALGORITHM & \multicolumn{2}{|c|}{ FEATURE } & \multicolumn{2}{|c|}{ ADVANTAGE } & \multicolumn{2}{|c|}{ DISADVANTAGE } \\
\hline $\begin{array}{l}\text { SIFT } \\
\text { (Scale Invariant } \\
\text { Feature } \\
\text { Transformation) }\end{array}$ & $\begin{array}{l}\text { I. } \\
\text { II. } \\
\text { III. } \\
\text { IV. } \\
\mathrm{V} .\end{array}$ & $\begin{array}{l}\text { Scale Space Extreme } \\
\text { Detection } \\
\text { Key point Localization } \\
\text { OrientationAssignment } \\
\text { Feature Description } \\
\text { Feature Matching }\end{array}$ & $\begin{array}{l}\text { I. } \\
\text { II. } \\
\text { III. } \\
\text { IV. } \\
\text { V. }\end{array}$ & $\begin{array}{l}\text { Locality } \\
\text { Distinctiveness } \\
\text { Quantity } \\
\text { Efficiency } \\
\text { Extensibility }\end{array}$ & II. & $\begin{array}{l}\text { Being a 128- } \\
\text { vector is } \\
\text { relatively slow to } \\
\text { compute and } \\
\text { match. } \\
\text { Generally doesn't } \\
\text { work well with } \\
\text { lighting changes } \\
\text { and if blur. }\end{array}$ \\
\hline $\begin{array}{l}\text { Surf } \\
\text { (Speeded Up Robust } \\
\text { Feature ) }\end{array}$ & $\begin{array}{l}\text { I. } \\
\text { II. } \\
\text { III. }\end{array}$ & $\begin{array}{l}\text { Exploit the integral image. } \\
\text { Creates a grid around the key } \\
\text { point. } \\
\text { Divides each grid cell into sub- } \\
\text { grids. }\end{array}$ & III. & $\begin{array}{l}\text { Reuse the calculations } \\
\text { Maintain the } \\
\text { robustness to rotation, } \\
\text { scale illumination } \\
\text { change. } \\
2 X \text { faster than } \\
\text { Difference of } \\
\text { Gaussian. } \\
\text { Improves on SIFT by } \\
\text { using a box filter } \\
\text { approximation. }\end{array}$ & $\begin{array}{l}\text { II. } \\
\text { III. } \\
\text { IV. }\end{array}$ & $\begin{array}{l}\text { Invariance } \\
\text { reduces the } \\
\text { ability to } \\
\text { discriminate. } \\
\text { Difficult to track } \\
\text { edges robustly. } \\
\text { View dependent. } \\
\text { More fragile }\end{array}$ \\
\hline
\end{tabular}

\section{CONCLUSION}

SURF based for the most part imitation identification algorithmic projects region unit bottomless speedier than SIFT based picture phony location calculation. The morphological operations give the higher outcomes to the recognition of change inside the cast half. The long run work centers to fabrication in video and grafting.

\section{REFERENCES}

[1] Jessica Fridrich, David Soukal, and Jan Lukas, " Detection of Copy-Move Forgery in Digital Images" , in Proc. Advanced Forensic Res.Works., Cleveland,OH, Aug. 2003.

[2] C. Popescu and H. Farid, "Uncovering digit. falsifications by distinguishing copied picture locales," Dept. Comput. Sci., Dartmouth College, Hanover, NH, USA, Tech. Rep. TR2004-515, 2004

[3] Saiqa Khan, Arun Kulkarni, "Strong strategy for location of Copy-Move Forgery in Digit. Pictures" International Conference on Signal and Image Processing,2010.
[4] L. Fitzpatrick and M.Dent, " Region Duplication Detection Using Image Feature Matching" ,Ieee Transactions On Information Forensics And Security, Vol. 5, No. 4,2010.

[5] Jian Li , Xiaolong Li ,Bin Yang and Xingming Sun, " Segmentation-Based Image Copy-Move Forgery Detection Scheme", IEEE Transactions on Information Forensics and Security, Volume:10, dec 2014.

[6] Irene Amerini, Lamberto Ballan, Roberto Caldelli, Alberto Del Bimbo, and Giuseppe Serra, "A SIFT Based Forensic Method for Copy-Move Attack Detection and Transformation Recovery" , Ieee Transactions On Information Forensics And Security, Vol. 6, No. 3, September 2011

[7] Chi-Man Pun, Xiao-Chen Yuan, and Xiu-Li Bi, "Picture Forgery Detection Using Adaptive Oversegmentation and Feature Point Matching", Ieee Transactions On Information Forensics And Security, Vol. 10, No. 8, August 2015 